\title{
NMR relaxometry as a potential non-invasive routine sensor for characterization of phenotype in Crassostrea gigas
}

\author{
Armel Davenel ${ }^{a, c,{ }^{*}}$, Stéphane Pouvreau ${ }^{b}$, Mireille Cambert ${ }^{a, c}$, Marc Suquet ${ }^{b}$ and François \\ Mariette ${ }^{a, c}$
}

\footnotetext{
${ }^{a}$ Cemagref, UR TERE, 17 avenue de Cucillé - CS 64427, F-35044 Rennes, France

${ }^{\mathrm{b}}$ Ifremer, UMR100, LPI, Station Expérimentale d'Argenton, 29840 Argenton en Landunvez, France

${ }^{\mathrm{c}}$ Université Européenne de Bretagne, France
}

*: Corresponding author : Armel Davenel, Tel.: +33 22348 2160; fax: +33 22348 2115, email address : armel.davenel@cemagref.fr

\begin{abstract}
:
MR imaging is the most appropriate non-invasive technique for quantifying the growth of somatic and gonad tissues and to determine sex in the Pacific oyster, Crassostrea gigas. However, this technique is too costly for field studies where oysters are used as bioindicators of environmental quality or to be applied routinely in hatcheries. We have tested the ability of low Nuclear Magnetic Resonance relaxometry, a much less expensive technique, to obtain phenotype parameters that can be used to monitor the physiological state of oysters. NMR measurements were carried out at three different periods using a low field spectrometer equipped with a $50 \mathrm{~mm}$ diameter probe to investigate 60 oysters in their first year of maturity, which were then dissected to measure internal shell cavity volume and dry flesh weight and to determine sex and gonad development. The NMR results showed that it was possible to determine both internal shell cavity volume and dry flesh weight in less than one minute with very high determination $R^{2}$ coefficients ( 0.95 and 0.94 , respectively). The results showed also that it was possible to identify sex and gonad development, with success rates of $93 \%$ and $83 \%$, respectively. For oysters with dry weight above $0.7 \mathrm{~g}$, the success rate in identifying sex was $100 \%$. Further studies are required to design an NMR probe that is appropriate for larger oysters and to improve sex discrimination and prediction of gonad development with larger study groups.
\end{abstract}

Keywords: Phenotype characterization; Crassostrea gigas; NMR; Growth; Sex identification 


\section{Introduction}

With an annual production of above 117,500 metric tons in 2006, production of the Pacific oyster Crassostrea gigas is economically important in French aquaculture. However, the ecology and physiology of this bivalve are not fully understood, and consequently monitoring of its growth and reproduction, both in the field and in hatcheries, is still based on empirical factors. Investigation of soft tissues in marine mollusks, especially in marine bivalves, classically relies on destructive methods, since a hermetic shell protects the animal. For example, anatomical structures are generally studied after opening and dissection by means of histological sections, with a resolution of 4-5 $\mu \mathrm{m}$ (Chavez-Villalba et al., 2002; Didri et al., 2007). The evolution of gametogenesis can be quantitatively assessed with the help of image analysis (Chavez-Villalba et al., 2003; Fabioux et al., 2005), and sex may be determined after anesthesia using magnesium chloride and tissue sampling using needles (Namba et al., 1995). However, analysis of physiological and biochemical changes requiring large volume tissue samples necessarily involves the sacrifice of numerous specimens and the preparation and analysis of several samples. These standard techniques provide valuable information on marine mollusk biology but have two main disadvantages which are limitations for many studies: these methods are very time consuming and they are necessarily destructive. Non-invasive and quantitative procedures have therefore been developed, and after preliminary trials (Pouvreau et al., 2006) Nuclear Magnetic Resonance (NMR) imaging (MRI) has proved promising.

Non-invasive characterization of gonad maturation and determination of the sex of Pacific oysters by Magnetic Resonance Imaging (MRI) has already been successfully tested using longitudinal relaxation T1-weighted MRI sequences (Davenel et al., 2006). MRI is the most appropriate technique for quantification of the growth of somatic and gonad tissues and to determine sex. However, this technique is too costly for field studies and particularly for routine use in hatcheries. Preliminary results (Davenel et al., 2006) of the characterization of various 
oyster tissue samples by NMR relaxometry, a much less expensive technique, showed that the T1 relaxation times of gonad and muscle were shorter than those of the heart, other viscera and gills which are bathed in seawater, itself characterized by very high $\mathrm{T} 1$ and $\mathrm{T} 2$ relaxation time values (more than $1600 \mathrm{~ms}$ ). These experiments ascertained that the NMR technique was able to differentiate the ovaries (T1 of about $207 \pm 21 \mathrm{~ms}$ ) from the testes (T1 of about $456 \pm 50 \mathrm{~ms}$ ) and muscles (T1 of about $461 \pm 13 \mathrm{~ms}$ ). However, the NMR technique did not make it possible to differentiate the male gonad and muscle on the basis of the T1 value alone. We report here the ability of NMR to obtain phenotype parameters that can be used to monitor the physiological state of entire oysters in their shells by multivariate data analysis techniques on NMR transverse $\mathrm{T} 2$ relaxation data weighted by longitudinal $\mathrm{T} 1$ relaxation using different relaxation delays.

\section{Materials and methods}

\subsection{Origin of animals and preparation}

NMR measurements were carried out at three different periods (May 7, June 4 and June 29 2007) on 31 diploid $(2 \mathrm{~N})$ and 25 triploid (3N) Crassostrea gigas oysters in their first year of maturity to limit their size to the $52 \mathrm{~mm}$ diameter of the NMR probe. They were bred by the IFREMER Shellfish Laboratory located in Argenton near Brest (Brittany, France) and brought to Cemagref in Rennes (Brittany, France) for NMR investigations without any specific anesthetization procedure (distance $250 \mathrm{~km}$ ). Before NMR measurements, animals were soaked in sea water to expel any air bubbles. Sea water was kept at room temperature with the addition of phytoplankton to facilitate the opening of oysters.

After NMR measurements, each oyster was measured and weighed using a standard method. First, individual total mass (i.e. shell plus tissue), was weighed to the nearest $0.1 \mathrm{~g}$ on an electronic Sartorius balance (TW). Oysters were then opened and the flesh was drained for 15 min on absorbent paper and weighed using an electronic Sartorius balance to the nearest $0.001 \mathrm{~g}$ to determine fresh flesh weight (FW). The difference between total weight (TW) and flesh weight (FW) provides an estimate of the internal shell cavity (ISC) expressed as mass unit (g). 
Gonad development (GD) was visually estimated at four qualitative stages from stage 0 (no visible gonad tissues) to stage 3 (well developed gonad tissues). A biopsy was then taken from each gonad and analyzed under a light microscope to determine sex. The presence of spermatozoa $(2-3 \mu \mathrm{m})$ or oocytes $(30-50 \mu \mathrm{m})$ in the sample indicated if oysters were male $(\mathrm{M})$ or female $(\mathrm{F})$, respectively. Triploid oysters that were asexual were annotated 3N. Finally dry flesh weight (DW) was measured after a 72-hour freeze-drying cycle.

\subsection{NMR measurements}

NMR measurements were performed at Cemagref (Rennes, Brittany, France) with an OXFORD MQA 6005 spectrometer operating at $0.12 \mathrm{~T}(5 \mathrm{MHz})$ equipped with a $52 \mathrm{~mm}$ vertical diameter probe which allowed investigation of oysters in their first year of maturity $(<45 \mathrm{~g}$ total weight). NMR data comprised the intensities of echoes originating from an NMR pulse sequence that acquired two Carr-Purcell-Meiboom-Gill (CPMG) spin echo trains with different relaxation delay (RD) times between each signal accumulation reflecting combined $\mathrm{T} 1$ and $\mathrm{T} 2$ relaxation times (Table 1). The $15000 \mathrm{~ms}$ RD time was chosen to obtain the full T2 relaxation signal of sea water in the internal shell cavity without any T1 weighting. The $400 \mathrm{~ms}$ RD time corresponded to the T1-weighting parameter used in previous MRI experimentations (Davenel et al., 2006) to obtain the best contrast between testes and ovaries. Low-field NMR measures the spin-spin (T2) and spin-lattice (T1) relaxation of hydrogen nuclei (protons) in mobile or less mobile molecules.

\subsection{Statistical analysis}

Ideally, after data treatment based on Levenberg-Marquardt or maximum entropy decomposition, low field NMR measurements lead to a distribution of spin-spin relaxation times with peaks corresponding to resonance states of hydrogen nuclei that may be related to specific classes of molecules in the sample, each class characterized by its state (liquid/solid), its diffusion rate or its type of bonding or interaction with other molecules. However, if the rate of chemical exchange of protons between different classes is faster than the time scale of the NMR experiment, two or more classes may be indistinguishable from each other and the exact number 
and types of classes cannot be measured. We therefore chose to use other approaches based on

112 chemometric techniques to extract information from the NMR CPMG relaxation curves.

Determination of quantitative phenotype characteristics (ISC, FW and DW).

114 Because the first echo intensity of the non-T1-Weighed CPMG sequence with long RD

$115(15000 \mathrm{~ms})$ is proportional to the total proton density of the sample, we tested the simple linear

116 relationship between ISC and the intensity of this variable, and also the intensity of the first echo

117 of the T1-Weighed CPMG sequence with shorter RD value (400ms). Protons in the flesh were

118 water protons interacting with macromolecules or were constituents of lipids and their T2

119 relaxation parameters were shorter than sea water protons. Consequently we tested the linear

120 relationship between flesh weight (FW) or dry weight (DW) and a combination of the intensity

121 of the first echo and the intensity of a longer echo time, strongly weighted by the transverse

122 relaxation of the sea water protons.

123 The traditional univariate linear and multiple regression methods are of limited value to handle

124 the large amount of co-linear data points acquired by CPMG sequences. Multivariate data

125 analysis (chemometric) techniques include algorithms that can handle large co-linear data

126 structures. In this context, the main advantage of chemometric tools is that they are able to deal

127 with spectral or relaxation information, such as NIR and NMR (multivariate co-linear data) by

128 reducing data to a few latent factors (LF). Correlations between quantitative phenotype and

129 NMR data were evaluated using the partial least squares (PLS) predictive regression method on

130 relaxation data originating from the CPMG sequence for each RD value (CPMG-400, CPMG-

131 15000) separately and in combination with NMR data (CPMG-400-15000).

132 Determination of qualitative phenotype characteristics (SEX, GD).

133 First non-supervised principal component analysis (PCA) was performed to reduce the

134 dimensions of the original CPMG data matrices retaining the maximum amount of variability.

135 This provided a new set of variables (principal components, PCs) which facilitated the discovery

136 of patterns hidden in the dataset. A stepwise linear discriminant analysis (LDA), a supervised 
137 method used for classification purposes, was then performed on PC variables. LDA renders a

138 number of orthogonal linear discriminant functions equal to the number of classes minus one.

139 This method maximizes variance between categories and minimizes variance within classes.

140 Three sex classes were defined: male (M), female (F) and triploid or asexual oysters (3N), and

141 the four qualitative stages described above were used for classification according to gonad

142 development of diploid oysters.

143 Stepwise PLS and LDA procedures were combined with the leave-one-out cross-validation 144 procedure to seek subsets of synthetic LF or PC variables that were the most useful to evaluate 145 predictive errors (RMSEP) of quantitative phenotype characteristics or to discriminate between 146 classes and estimate the clearly classified percentages of qualitative characters. All of the 147 chemometrics discussed were performed using the statistical package $\mathrm{R}$ ( $\mathrm{R}$ Foundation for 148 Statistical Computing, Vienna, 2006: www.R-project.org).

\section{Results}

\subsection{Determination of quantitative phenotype characteristics (ISC, FW and DW).}

In terms of the relationships between the quantitative phenotype characteristics themselves, measured by the standard destructive methods, it should be noted that fresh weight (FW) and dry

154 weight (DW) were highly correlated $\left(\mathrm{R}^{2}: 0.94\right)$, demonstrating the good repeatability of the 155 method used to measure fresh weight. Internal shell cavity (ISC) volume was moderately 156 correlated with FW and DW ( $\left.\mathrm{R}^{2}: 0.56\right)$.

157 Simple univariate correlation between internal cavity volume (ISC) and the intensity of the first 158 echo (echo1) of the CPMG-15000 relaxation curve showed that it was possible to determine ISC 159 with a very high correlation coefficient ( $\mathrm{R}^{2}$ of 0.95$)$. In the range $4.7 \mathrm{~g}$ to $16 \mathrm{~g}$, ISC was predicted 160 with a standard error of prediction of $0.62 \mathrm{~g}$ (Table 2). 
161 To eliminate the influence of the sea water protons on the NMR signal, slightly more

162 sophisticated relationships were necessary to predict FW and DW. The results showed that the 163 best combination was to subtract the intensity of a spin echo strongly affected by sea water 164 protons from the first spin echo of the relaxation curve. All the possible combinations were 165 tested. After optimization, the value resulting from the subtraction of the intensity of the echo at $16664 \mathrm{~ms}$ from the beginning of the relaxation curve from the first echo of the CPMG-400 data 167 appeared to be the best correlated with DW and FW, with correlation coefficients of 0.94 and 1680.91 , respectively. In the range $0.22 \mathrm{~g}$ to $2.10 \mathrm{~g}$, DW was predicted with a standard error of 169 prediction of less than $0.1 \mathrm{~g}$ (Table 2, figure 1). FW was predicted with a standard error of 170 prediction of less than $0.54 \mathrm{~g}$ in the range $1.28 \mathrm{~g}$ to $8.90 \mathrm{~g}$ (Table 2).

171 Several combinations of latent variables resulting from the PLS procedure showed good 172 correlations but did not provide a very significant improvement in the relationships with the 173 different quantitative phenotype characteristics such as DW and FW despite the combinations of

1745 or 6 latent variables (Table 3). However, the combination of only two latent variables resulting 175 from the CPMG-400 curves alone provided a potentially acceptable prediction of ISC ( $\mathrm{R}^{2}$ : 0.93 ; 176 RMSEP: $0.78 \mathrm{~g})$.

1773.2 Determination of qualitative phenotype characteristics (SEX, GD).

178 Stepwise linear discriminant analysis (LDA) of each CPMG curve result separately showed that 179 SEX could be classified with a success rate of $59 \%$ and $71 \%$ with CMPG-400 and CPMG180 15000, respectively. Based on a combination of the results from both CPMG curves, LDA 181 showed a very significant increase in the success rate (to 93\%) to predict SEX (Table 4). 182 Discriminant function 1, explaining $78 \%$ of the variance, could be interpreted as well correlated 183 with ploidy, and discriminant function 2 clearly separated $2 \mathrm{~N}$ males and females (Figure 2).

184 Stepwise linear discriminant analysis based on the combination of data from both CPMG curves 185 showed that the potential of NMR relaxometry to discriminate gonad development was good, 186 with $84 \%$ of $2 \mathrm{~N}$ oysters correctly classified (Table 5 ). 


\section{Discussion}

188 These preliminary results show that the low field NMR relaxometry technique is a potentially

189 non-invasive routine sensor for phenotype characterization in Crassostrea gigas. The technique

190 provides access to two important numerical representations of the quality of soft bivalve tissue:

191 the weight of the soft tissue and the meat condition index based on the percentage of the internal

192 shell volume occupied by soft body tissue. The most promising result was the estimation of the

193 fresh or dried soft tissue weight by simple bilinear regression based on the CPMG-400 curve,

194 obtained in less than a minute. Using $400 \mathrm{~ms}$ RD time, the weight of the very mobile hydrogen

195 atoms (protons) in the CPMG-400 relaxation signal was considerably reduced compared to less

196 mobile protons of macromolecules constituting the dry soft tissue matter, characterized by a

197 longitudinal relaxation T1 parameter that was shorter than for seawater protons. Seawater

198 protons were also characterized by a longer transverse T2 relaxation parameter: subtraction of

199 the intensity of a longer echo time, such as the $64 \mathrm{~ms}$ echo time, strongly weighted by the sea

200 water protons from the intensity of the first echo time, provided an indicator that was correlated 201 with the quantity of protons in the macromolecules of the dry soft tissue matter. However, Figure

2 shows that the dry weight of oysters with high gonad development could be slightly underestimated. This could be related to the effects of differences in the biochemical composition of soft tissue: gonad tissues are high in lipids (Matus de la Parra et al. 2003) which have higher proton density than glycogen and proteins. Clearly this method would be less precise than the gravimetric method based on lyophilization, but the new method has the advantage of being fast and easy to use for routine investigations and its non-destructive nature should allow precise individual follow-up studies of large oyster collections.

The NMR technique also appears to be able to provide an acceptable determination of ISC that

210 can be used to calculate a condition index. ISC is clearly correlated with the intensity of the first 211 echo of the non-weighted CMPG-15000 relaxation curve which indicates the quantity of all the 212 protons from soft tissues and seawater in the NMR probe. The main difficulty is being sure that 
213 the internal shell cavity volume is full of seawater and that shell valves remain closed during

214 NMR scanning. An NMR sensor with a horizontal probe would be more appropriate to reduce

215 the risk of water loss. Early methods sought to measure the internal shell cavity by volumetric

216 methods but these are slow and difficult to perform accurately. A faster and easier gravimetric

217 method was also developed: shell cavity capacity was well correlated with the difference

218 between whole oyster weight and empty shell weight after drying for $24 \mathrm{~h}$ (Laurence and Scott,

219 1982). However, with this method, any water contained within the shells themselves (not

220 between them) was included in the internal shell cavity weight (Abbe and Albright, 2003). The

221 same limitation exists with the NMR method which quantifies all the protons contained in the

222 NMR probe. Differences in shell morphology and fouling community structure may influence

223 shell porosity and reduce the precision of the method.

224 Exploitation of all the data originating from both CMPG relaxation curves by more sophisticated

225 chemometric methods provided encouraging results that also showed the good potential of NMR

226 to determine sex and identify gonad development. However, given the small number of

227 individuals and the difficulty in visually appreciating gonad development, further studies with

228 larger sample groups are required to validate the method. LDA performed on individuals with

229 dry tissues weighing over $0.7 \mathrm{~g}$ provided a success rate of $100 \%$. This clearly shows that the

230 precision of the NMR method would be lower for small individuals. The diameter of the NMR

231 probe used in this study did not allow scanning of larger oysters, particularly oysters in their

232 second or third year of maturity. Further studies are required to design an NMR probe that is

233 appropriate for bigger oysters and to improve sex discrimination and prediction of gonad

234 development with larger groups. The method to determine soft tissue weight is rapid and

235 probably sufficiently precise to investigate even small animals. The determination of internal

236 shell cavity, sex and gonad development requires the acquisition of a complementary NMR

237 signal based on a CMPG sequence with a long RD parameter. At the present time, this increases

238 the acquisition time by 7 minutes. It would probably be advisable to reduce the overall 
acquisition time by half and it should be possible to replace the non-T1-weighted CPMG-15000

240 sequence by a slightly T1 weighted sequence with RD between 5000 and $8000 \mathrm{~ms}$ to achieve this.

241 Another possibility would be to reduce the accumulation number (16 in the present study),

242 particularly for the largest oysters which deliver the strongest signal originated from soft tissues.

5. References

Abbe, G. R., Albright, B. W., 2003. An improvement to the determination of meat condition index for the eastern oyster Crassostrea virginica (Gmelin 1791). J Shellfish Res 22:747752.

Davenel, A., Quellec, S., Pouvreau, S. 2006. Noninvasive characterization of gonad maturation and determination of the sex of Pacific oysters by MRI. Magnetic Resonance Imaging 24 (8), 1103-1110.

Dridi, S., Salah Romdhane, M., Elcafsi, M. 2007. Seasonal variation in weight and biochemical composition of the Pacific oyster, Crassostrea gigas in relation to the gametogenic cycle and environmental conditions of the Bizert lagoon, Tunisia. Aquaculture 263 (1-4), 238248.

Chavez-Villalba, J., Pommier, J., Andriamiseza, J., Pouvreau, S., Barret, J., Cochard, J.C., Le Pennec, M., 2000. Broodstock conditioning of the oyster Crassostrea gigas: origin and temperature effect. Aquaculture 214 (1-4), 115-130.

Enriquez-Diaz, M., Pouvreau S., Fabioux, C., Le Coguic, Y., Cochard, J.C., Le Pennec, M., 2003. Reproductive strategy: Variability of reproductive pattern in two populations of genetically determined Crassostrea gigas. J Shellfish Res 22:328.

Fabioux, C., Huvet, A., Le Souchu, P., Le Pennec, M., Pouvreau, S., 2005. Temperature and photoperiod drive Crassostrea gigas reproductive internal clock. Aquaculture 250 (1-2), 458-470.

Lawrence, D. R., Scott, G.I., 1982. The determination and use of condition index in oysters. Estuaries 5:23-27.

Matus de la Parra, A., Garcia, O., San Juan, F., 2005. Seasonal variations on the biochemical composition and lipid classes of the gonad and storage tissues of Crassostrea gigas (Thuberg, 1794) in relation to the gametogenic cycle. J Shellfish Res 24 (2):457-467.

Namba K., Kobayashi M., Aida S., Uematsu K., Yoshida M., Kondo Y., Miyata Y., 1995. 

magnesium ion. Fish. Sci. 61, 241-244.

273 Pouvreau, S., Rambeau, M., Cochard, J.C., Robert, R., 2006. Investigation of marine bivalves 274 morphology by in vivo MR imaging: first anatomical results of a promising technique. Aquaculture 259 (1-4), 415-423.

276 


\section{Captions for figures}

279 Fig.1. Prediction of dry flesh weight by subtraction of the intensity of the echo at $64 \mathrm{~ms}$ from the beginning of the CPMG-400 relaxation curve from the intensity of the first echo. Effects of gonad development stages $(0,1,2$, and 3$)$. $3 \mathrm{~N}$ individuals were classified as stage 0 .

282 Fig.2. Prediction of sex (3N triploid asexual oysters, $2 \mathrm{~N}$ males and females) by discriminant 283 functions originating from chemometric LDA method.

284

285

286 
Table 1. Parameters of $\mathbf{N M ~ R}$ sequences

\begin{tabular}{ccccc}
\hline $\begin{array}{c}\text { NM R } \\
\text { CPM G sequence }\end{array}$ & RD & TE & $\begin{array}{c}\text { Accumulation } \\
\text { number }\end{array}$ & $\begin{array}{c}\text { Acquisition } \\
\text { time }\end{array}$ \\
\hline CPM G-400 & $15000 \mathrm{~ms}$ & $2 \mathrm{~ms}$ & 16 & $6 \mathrm{~min} 51 \mathrm{~s}$ \\
CPM G-15000 & $400 \mathrm{~ms}$ & $1 \mathrm{~ms}$ & 32 & $47 \mathrm{~s}$ \\
\hline
\end{tabular}


Table 2. Prediction of quantitative phenotype characteristics, dry flesh weight (DW), fresh flesh weight (FW) and internal shell cavity (ISC) using simple or bilinear relationship with NM R data.

\begin{tabular}{llllllll}
\hline NM R data & Echo and & \multicolumn{3}{c}{ DW } & \multicolumn{2}{l}{ FW } & \multicolumn{1}{l}{ ISC } \\
\cline { 3 - 8 } & Echo combination & $\mathrm{R}^{2}$ & RM SEP & $\mathrm{R}^{2}$ & RMSEP & $\mathrm{R}^{2}$ & RM SEP \\
\hline CPM G-400 & echo1 & 0.78 & & 0.83 & & 0.89 & $0.92 \mathrm{~g}$ \\
& echo1-echo64 & 0.94 & $0.100 \mathrm{~g}$ & 0.91 & 0.54 & & \\
CPM G-15000 & echo1 & 0.44 & & 0.53 & & 0.95 & $0.62 \mathrm{~g}$ \\
& echo1-echo34 & 0.91 & 0.116 & 0.89 & 0.56 & & \\
\hline
\end{tabular}

RM SEP means R oot M ean Square E rror of Prediction 
Table 3. Prediction of quantitative phenotype characteristics, dry flesh weight (DW), fresh flesh weight (FW) and internal shell cavity (ISC) using latent variables (LV) originating from chemometric PLS method

\begin{tabular}{|c|c|c|c|c|c|c|c|}
\hline \multirow[t]{2}{*}{ NM R data } & \multirow{2}{*}{$\begin{array}{l}\text { L atent } \\
\text { V ariables }\end{array}$} & \multicolumn{2}{|c|}{ DW } & \multicolumn{2}{|c|}{ FW } & \multicolumn{2}{|c|}{ ISC } \\
\hline & & $R^{2}$ & RM SEP & $R^{2}$ & RM SEP & $R^{2}$ & RM SEP \\
\hline CPM G-400 & $\begin{array}{l}3 \mathrm{LV} \\
2 \mathrm{LV}\end{array}$ & 0.96 & $0.093 \mathrm{~g}$ & 0.94 & $0.52 \mathrm{~g}$ & 0.93 & $0,78 \mathrm{~g}$ \\
\hline CPM G-15000 & $\begin{array}{l}6 \mathrm{LV} \\
5 \mathrm{LV}\end{array}$ & 0.95 & $0.116 \mathrm{~g}$ & 0.92 & $0.58 \mathrm{~g}$ & 0.98 & $0.67 \mathrm{~g}$ \\
\hline CPM G-400-15000 & $\begin{array}{l}5 \mathrm{LV} \\
5 \mathrm{LV}\end{array}$ & 0.96 & $0.110 \mathrm{~g}$ & 0.94 & $0.53 \mathrm{~g}$ & 0.99 & $0.65 \mathrm{~g}$ \\
\hline
\end{tabular}

RM SEP means Root M ean Square Error of Prediction 
Table 4. Prediction of sex ( $3 \mathrm{~N}, 2 \mathrm{~N}$ males and females) using discriminant functions originating from chemometric LDA method.

\begin{tabular}{ccccc}
\hline \multirow{2}{*}{ «Sex » observed } & \multirow{2}{*}{$\mathrm{n}$} & $\mathrm{3}$ & $\mathrm{F}$ & $\mathrm{M}$ \\
\cline { 3 - 5 } & & $\mathbf{2 5}(100 \%)$ & 0 & 0 \\
\hline $\mathrm{NN}$ & 25 & 0 & $\mathbf{1 4}(93 \%)$ & 1 \\
$\mathrm{~F}$ & 15 & 1 & 2 & $\mathbf{1 3}(87 \%)$ \\
$\mathrm{M}$ & 16 & & & \\
\hline
\end{tabular}


Table 5. Prediction of gonad development (GD) stages $(1,2,3)$ of $2 \mathrm{~N}$ males and females using discriminant functions originating from chemometric LDA method

\begin{tabular}{ccccc}
\hline GD observed & $\mathrm{n}$ & \multicolumn{3}{c}{ GD predicted } \\
\cline { 3 - 5 } & 2 & $\mathbf{1}(50 \%)$ & $1(50 \%)$ & 3 \\
\hline 1 & 10 & 0 & $\mathbf{7}(70 \%)$ & $3(30 \%)$ \\
2 & 19 & 0 & $4(20 \%)$ & $\mathbf{1 8 ( 7 9 \% )}$ \\
3 & &
\end{tabular}


Fig.1.

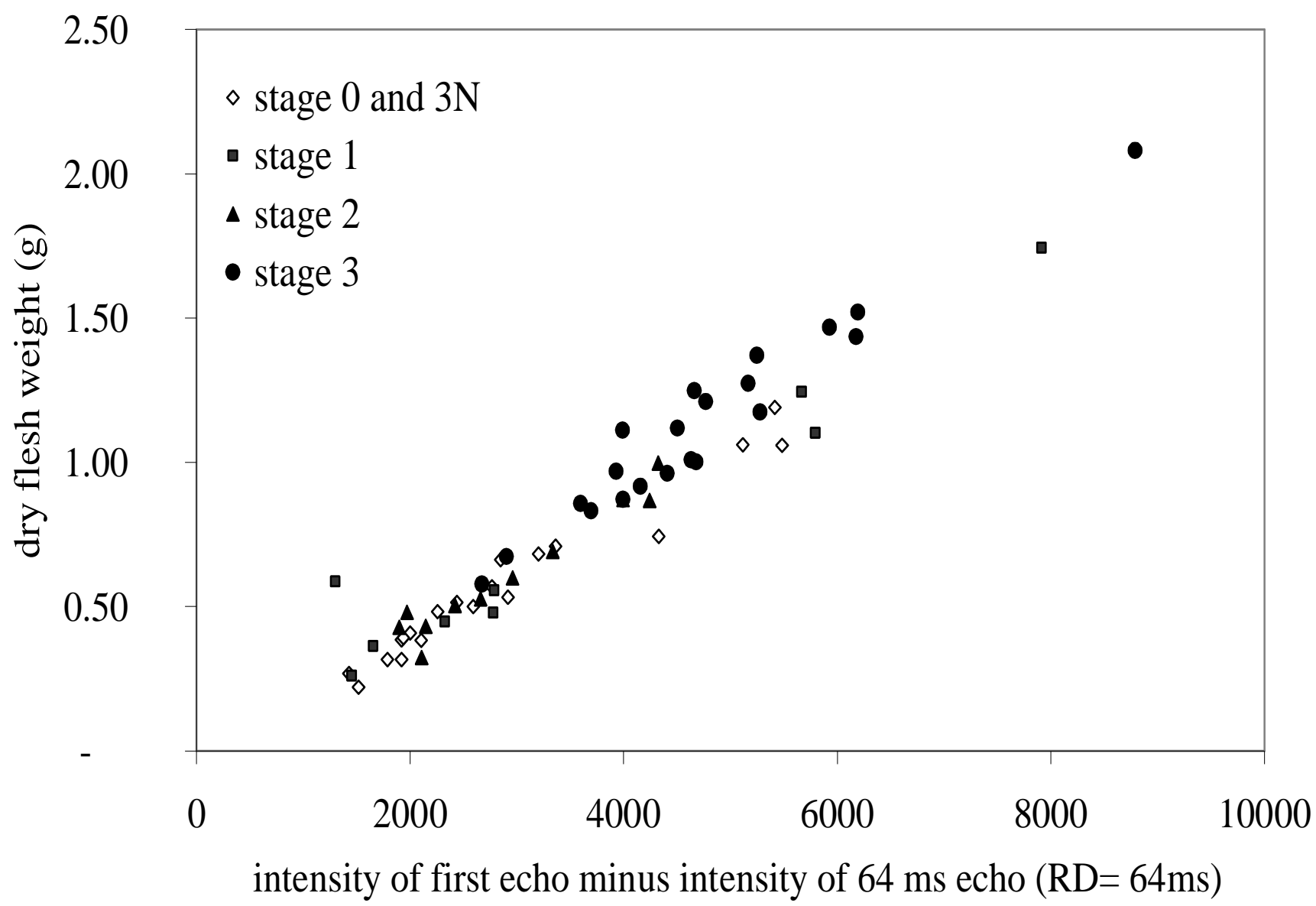


Fig.2.

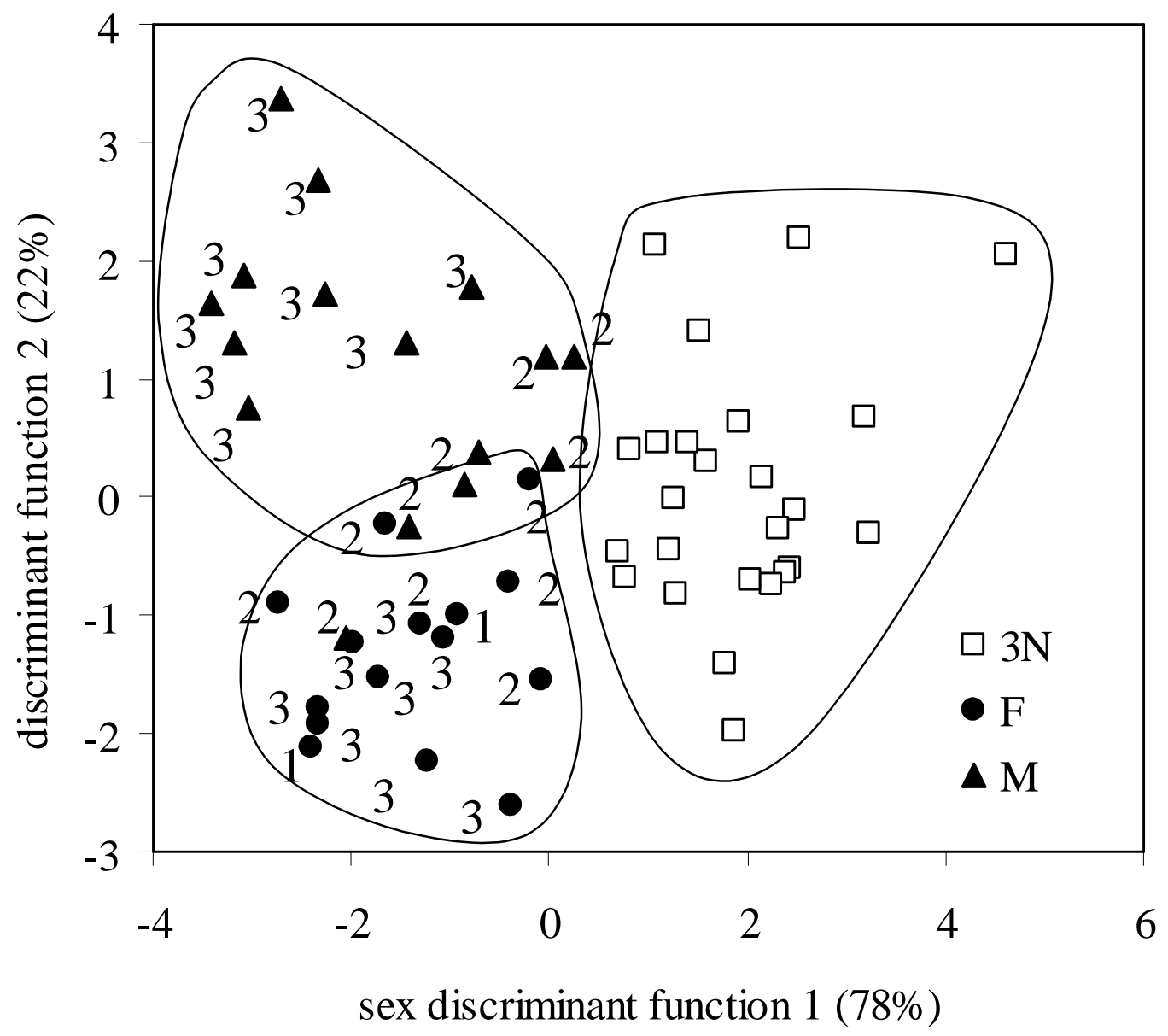




\begin{tabular}{|l|l|}
\hline \multicolumn{2}{|c|}{ M anuscript : N M R relaxometry as a potential non-invasive routine sensor for } \\
characterization of phenotype in Crassostrea gigas, A. Davenel...
\end{tabular}

